(April 24, 2002)

\title{
PATH INTEGRALS FOR CONTINUOUS-TIME MARKOV CHAINS
}

\author{
P.K. POLLETT, ${ }^{*}$ University of Queensland \\ V.T. STEFANOV, ${ }^{* *}$ University of Western Australia
}

\begin{abstract}
This note presents a method of evaluating the distribution of a path integral for Markov chains on a countable state space. We illustrate the method with reference to several models, including birth-death processes and the birth, death and catastrophe process.

HITTING TIMES; EXTINCTION TIMES; POPULATION PROCESSES.

AMS 2000 SUBJECT CLASSIFICATION: PRIMARY 60J27

SECONDARY $60 \mathrm{~J} 80$
\end{abstract}

\section{Introduction}

Let $(X(t), t \geq 0)$ be a continuous-time Markov chain taking values in the non-negative integers $S=\{0,1, \ldots\}$ and let $A$ be a fixed subset of $S$. Consider the path integral

$$
\Gamma=\int_{0}^{\tau} f_{X(t)} d t
$$

where $f: A \rightarrow[0, \infty)$ and $\tau=\inf \{t>0: X(t) \notin A\}$ is the first exit time of $A$. Here $f_{j}$ may be regarded as the reward per unit time of staying in state $j$, so that $\Gamma$ is the total reward over the period that the chain spends in $A$. We shall describe a method of obtaining the Laplace transform of the distribution of $\Gamma$, which is a simple extension of the corresponding result ( $f$ identically 1 ) on the distribution of first passage times (Syski $[13]$ ). Explicit results are available for special Markov chains. For example, when the state space is finite, the distribution of the time spent in $A$ can be exhibited explicitly (see Rubino and Sericola [11]). In the case of birth-death processes, Flajolet and Guillemin [6], and Ball and Stefanov [3], obtained results on transforms of the distributions of first passage times, and other fundamental characteristics, in terms of continued fractions.

\section{Distribution of the path integral}

Let $Q=\left(q_{i j}, i, j \in S\right)$ be the $q$-matrix of transition rates of the chain (assumed to be stable and conservative), so that $q_{i j}$ represents the rate of transition from state $i$ to state $j$, for $j \neq i$, and $q_{i i}=-q_{i}$, where $q_{i}:=\sum_{j \neq i} q_{i j}(<\infty)$ represents the total rate out of state $i$.

\footnotetext{
* Postal address: Department of Mathematics, The University of Queensland, Queensland 4072, Australia. E-mail address: pkp@maths.uq.edu.au

** Postal address: Department of Mathematics and Statistics, The University of Western Australia, Crawley (Perth), Western Australia 6009, Australia. E-mail address: stefanov@maths.uwa.edu.au
} 
It will not be necessary to assume that $Q$ is regular, so that there may actually be many processes with the given set of rates. However, we will take $(X(t), t \geq 0)$ to be the minimal chain associated with $Q$.

We shall evaluate the Laplace transform of the distribution of path integral (1), conditional on the chain starting in state $i \in A$, making the harmless assumption that $q_{j}>0$, for all $j \in$ $A$, so that $A$ contains no absorbing states. Let $y_{i}(\theta)=\mathrm{E}_{i}\left(e^{-\theta \Gamma}\right)$, with the understanding that $y_{i}(\theta)=1$ when $i \notin A$. (Here and henceforth we will use the notation $\mathrm{E}_{i}(\cdot)=\mathrm{E}(\cdot \mid X(0)=i)$ and $\mathrm{P}_{i}(\cdot)=\operatorname{Pr}(\cdot \mid X(0)=i)$.) The following result is a simple extension of the standard characterization of hitting times (for example, Theorem 9 on Page 86 of Syski [13]).

Proposition 1. For each $\theta>0, y(\theta)=\left(y_{i}(\theta), i \in S\right)$ is the maximal solution to the system

$$
\sum_{j \in S} q_{i j} z_{j}=\theta f_{i} z_{i}, \quad i \in A,
$$

with $0 \leq z_{j} \leq 1$ for $j \in A$ and $z_{j}=1$ for $j \notin A$, in the sense that $y(\theta)$ satisfies these equations, and, if $z=\left(z_{i}, i \in S\right)$ is any such solution, then $y_{i}(\theta) \geq z_{i}$ for all $i \in S$.

Proof. Fix $i \in A$ and condition on the time of the first jump and the state visited at that time, to get

$$
\mathrm{E}_{i}\left(e^{-\theta \Gamma}\right)=\int_{0}^{\infty} \sum_{k \neq i} e^{-\theta f_{i} u} \mathrm{E}_{k}\left(e^{-\theta \Gamma}\right) \frac{q_{i k}}{q_{i}} q_{i} e^{-q_{i} u} d u
$$

It follows that

$$
\sum_{k \neq i} q_{i k} y_{k}(\theta)=\left(\theta f_{i}+q_{i}\right) y_{i}(\theta)
$$

and we deduce immediately that $y(\theta)$ satisfies $(2)$.

To show that $y(\theta)$ is the maximal solution to $(2)$, let $T_{j}(n), n \geq 1$, be the total time that the process spends in state $j$ during the period up to the time of the $n^{\text {th }}$ jump, and let $y_{i}(n, \theta)=\mathrm{E}_{i}\left(e^{-\theta \Gamma(n)}\right)$, where $\Gamma(n)=\sum_{j \in S} f_{j} T_{j}(n)$, with the understanding that $y_{i}(n, \theta)=1$ for $i \notin A(\Gamma(n)$ is the contribution to the path integral over the period). Clearly $\Gamma(n) \uparrow \Gamma$, so that monotone convergence gives $y_{i}(n, \theta) \downarrow y_{i}(\theta)$. Again fix $i \in A$. Using a similar conditioning argument, we find that, for any $n \geq 1$,

$$
\mathrm{E}_{i}\left(e^{-\theta \Gamma(n+1)}\right)=\int_{0}^{\infty} \sum_{k \neq i} e^{-\theta f_{i} u} \mathrm{E}_{k}\left(e^{-\theta \Gamma(n)}\right) \frac{q_{i k}}{q_{i}} q_{i} e^{-q_{i} u} d u
$$

and we deduce that

$$
\sum_{k \neq i} q_{i k} y_{k}(n, \theta)=\left(\theta f_{i}+q_{i}\right) y_{i}(n+1, \theta), \quad n \geq 1 .
$$

But, we also have $y_{i}(1, \theta)=q_{i} /\left(\theta f_{i}+q_{i}\right)$, and so (4) is valid for $n=0$ provided we put $y_{k}(0, \theta)=1$ for all $k \in S$. Now suppose that $z=\left(z_{j}, j \in S\right)$ is any solution to (2) with $0 \leq z_{j} \leq 1$ for $j \in A$ and $z_{j}=1$ for $j \notin A$, so that, in particular,

$$
\sum_{k \neq i} q_{i k} z_{k}=\left(\theta f_{i}+q_{i}\right) z_{i}, \quad i \in A .
$$


For $i \notin A$, we have $y_{i}(\theta)=z_{i}=1$. On comparing (4) and (5), we see that if $y_{i}(n, \theta) \geq z_{i}$, $i \in A$, for any fixed $n \geq 0$, we will then also have $y_{i}(n+1, \theta) \geq z_{i}, i \in A$. But, $z_{i} \leq 1=$ $y_{i}(0, \theta)$, and so, by mathematical induction, $y_{i}(n, \theta) \geq z_{i}, i \in A$, for every $n \geq 0$. Letting $n \rightarrow \infty$ shows that $y_{i}(\theta) \geq z_{i}, i \in A$, and this completes the proof.

Formal differentiation of (3) suggests a corresponding result on the expected value of the path integral, conditional on the chain starting in state $i \in A$. In fact, using similar arguments, we can arrive at the following result, which is a simple extension of Theorem 10 on Page 86 of Syski [13].

Proposition 2. $e=\left(e_{i}, i \in A\right)$, where $e_{i}=\mathrm{E}_{i}(\Gamma)$, is the minimal non-negative solution to the system

$$
\sum_{j \in A} q_{i j} z_{j}+f_{i}=0, \quad i \in A
$$

Remarks. If we set $f_{i}=1$ for all $i \in A$, then $\Gamma=\tau$, and so the above results can be used to determine the distribution and the expectation of $\tau$. In the case when $Q$ is regular, these reduce to well known and widely used results on hitting times; see Section 9.2 of Anderson [2].

Of particular interest are the cases (i) $A=S$ with $S$ irreducible, and (ii) $S=A \cup\{0\}$, with $A$ irreducible and 0 being an absorbing state that is accessible from $A$. In both cases, $\Gamma$ counts the reward over the lifetime of the chain. Note that if $Q$ is not regular, then, in case (i), $\tau$ is the explosion time of the chain (which is almost surely finite for all starting states). The above results might therefore be useful in biological applications, where we may wish to account for explosive behaviour by allowing the chain to perform infinitely-many transitions in a finite time. Case (ii) was considered by Stefanov and Wang [12] for birthdeath processes. They derived an explicit expression for the expectation $\mathrm{E}_{i}(\Gamma)$, building on earlier work of Hernández-Suárez and Castillo-Chavez [8], who studied the case $i=1$ and $f_{j}=j$.

On dividing equation (2) by $f_{i}$, we see that, conditional on $X(0)=i, \Gamma$ has the same distribution as $\tau$ for the Markov chain with transition rates $Q^{*}=\left(q_{i j}^{*}, i, j \in S\right)$ given by $q_{i j}^{*}=q_{i j} / f_{i}$ for all $i \in A$ such that $f_{i}>0$, and $q_{i j}^{*}=q_{i j}$ otherwise. This was observed for birth-death processes by McNeil [9]. It is intuitively reasonable. If $T_{j}$ is the total time that the process spends in state $j$ during the period up to time $\tau$, and $N_{j}$ is the number of visits to $j$ during that period, then $\Gamma=\sum_{j \in A} f_{j} T_{j}$ and $T_{j}=\sum_{n=1}^{N_{j}} X_{j n}$, where $\left\{X_{j n}, n=1,2, \ldots\right\}$ are iid exponential random variables with parameter $q_{j}$. Since the distribution of $N_{j}$ does not depend on the holding times, but rather on the transition probabilities $p_{i j}=q_{i j} / q_{i}$ of the jump chain, then, for states $j$ with $f_{j}>0, f_{j} T_{j}$ has the same distribution as the sum of $N_{j}$ iid exponential random variables with parameter $q_{j} / f_{j}$. Therefore, since (in an obvious notation) $p_{i j}^{*}=p_{i j}$, and $q_{i}^{*}=q_{i} / f_{i}$ for all $i \in A$ such that $f_{i}>0$, we would expect $\Gamma$ to have the same distribution as $\tau$ for the modified chain. This observation will be useful in studying specific models for which the distribution and the expectation of $\tau$ are known in sufficient generality to accommodate state-dependent transition rates. For example, in the case of birth-death processes, there are explicit expressions for the expected value of 
various hitting times, and, expressions for transforms of their distributions are available in terms of continued fractions (Flajolet and Guillemin [6], Ball and Stefanov [3]), while in several special cases the hitting time densities are known explicitly (see, for example, Di Crescenzo [5]).

\section{Some applications}

In this section we give several applications to specific models. We start with the simplest Markov chain that can exhibit explosive behaviour.

The pure-birth process. This process has $q_{i, i+1}=q_{i}>0, i \geq 0$, with all other transition rates equal to 0 . The minimal chain has a lifetime $\tau$, which is almost surely finite for all starting states if and only if the series $\sum_{j=0}^{\infty} 1 / q_{j}$ converges. Equations (2) and (6) have explicit solutions. From (6) we get $\mathrm{E}_{i}(\Gamma)=\sum_{j=i}^{\infty} f_{j} / q_{j}$. From (2) we get $\mathrm{P}_{i}(\Gamma<\infty)=1$ for all $i \in S$ if and only if $\mathrm{E}_{0}(\Gamma)<\infty$, in which case

$$
\mathrm{E}_{i}\left(e^{-\theta \Gamma}\right)=\prod_{j=i}^{\infty} \frac{q_{j}}{q_{j}+\theta f_{j}}, \quad \theta>0, i \in S .
$$

Birth-death processes. These have $q_{i, i+1}=a_{i}, i \geq 0, q_{i, i-1}=b_{i}, i \geq 1, q_{0}=a_{0}$ and $q_{i}=a_{i}+b_{i}, i \geq 1$, with all other transition rates equal to 0 . We will assume that the birth rates $\left(a_{i}, i \geq 0\right)$ and the death rates $\left(b_{i}, i \geq 1\right)$ are all strictly positive, except perhaps $a_{0}$, which might be 0. Thus, we can accommodate the two cases referred to above: if $a_{0}>0$, then $S$ is irreducible; otherwise, $S=A \cup\{0\}$, with $A=\{1,2, \ldots\}$ irreducible and 0 an absorbing state that is accessible from $A$.

Proposition 2 can be used to obtain explicit formulae for the expected value of the path integral. It is easy to show that, when $S$ is irreducible,

$$
\mathrm{E}_{i}(\Gamma)=\sum_{j=i}^{\infty} \frac{1}{\lambda_{j} \pi_{j}} \sum_{k=0}^{j} f_{k} \pi_{k}, \quad i \geq 0,
$$

where the potential coefficients $\left(\pi_{j}, j \geq 0\right)$ are given by $\pi_{0}=1$ and $\pi_{i}=\prod_{j=1}^{i} \lambda_{j-1} / \mu_{j}$, for $i \geq 1$. When 0 is an absorbing state, we need to distinguish two cases depending on whether or not the series $A=\sum_{i=1}^{\infty} 1 /\left(\mu_{i} \pi_{i}\right)$ converges, where now $\left(\pi_{j}, j \geq 1\right)$ are defined by $\pi_{1}=1$ and $\pi_{i}=\prod_{j=2}^{i} \lambda_{j-1} / \mu_{j}$, for $i \geq 2$. When $A=\infty$, a condition that corresponds to the process being non-explosive with absorption probability 1 , we have

$$
\mathrm{E}_{i}(\Gamma)=\sum_{j=1}^{i} \frac{1}{\mu_{j} \pi_{j}} \sum_{k=j}^{\infty} f_{k} \pi_{k}, \quad i \geq 1,
$$

this being finite if and only if $\sum_{k=1}^{\infty} f_{k} \pi_{k}<\infty$. When $A<\infty$, we have

$$
\mathrm{E}_{i}(\Gamma)=\sum_{j=1}^{i} \frac{1}{\mu_{j} \pi_{j}}\left(\frac{C(f)}{A}-\sum_{k=1}^{j-1} f_{k} \pi_{k}\right), \quad i \geq 1,
$$


where

$$
C(f)=\sum_{j=2}^{\infty} \frac{1}{\mu_{j} \pi_{j}} \sum_{k=1}^{j-1} f_{k} \pi_{k}=\sum_{j=1}^{\infty} \frac{1}{\lambda_{j} \pi_{j}} \sum_{k=1}^{j} f_{k} \pi_{k},
$$

with $\mathrm{E}_{i}(\Gamma)$ being finite if and only if $C(f)<\infty$.

Proposition 1 can be used to identify the distribution of $\Gamma$ for specific cases. To illustrate this, consider the linear birth-death process, which has $a_{i}=a i$ and $b_{i}=b i$ for $i \geq 0$, where $a$ and $b$ are positive constants. Suppose that $a<b$, so that the time $\tau$ to absorption is finite with probability 1 for all starting states, and consider the path integral $\Gamma=\int_{0}^{\tau} X(t) d t$. With this specification, equations (2) can be solved explicitly, and it is a simple matter to identify the minimal solution. We find that $\mathrm{E}_{i}\left(e^{-\theta \Gamma}\right)=(\gamma(\theta))^{i}$, where $\gamma(\theta)$ is the smaller of the two zeros of $a s^{2}-(a+b+\theta) s+b$ (which are both real and positive). Indeed, the Laplace transform can be inverted to give the probability density

$$
d \mathrm{P}_{i}(\Gamma \leq t)=\frac{i}{t} e^{-(a+b) t}\left(\frac{b}{a}\right)^{i / 2} I_{i}(2 t \sqrt{a b}) d t
$$

where $I_{i}(z)$ is the usual modified Bessel function of the first kind. This accords with the first-passage time density of state 0 for the $M / M / 1$ queue (Abate, Kijima and Whitt [1]); see the remark at the end of the previous section.

The birth-death and catastrophe process. This process, which was first studied by Brockwell [4], extends the linear birth-death process by allowing for downward jumps of arbitrary size (catastrophes). It has transition rates given by

$$
q_{i j}= \begin{cases}i \rho a, & i \geq 0, j=i+1, \\ -i \rho, & i \geq 0, j=i, \\ i \rho d_{i-j}, & i \geq 2,1 \leq j<i, \\ i \rho \sum_{k \geq i} d_{k}, & i \geq 1, j=0,\end{cases}
$$

with all other transition rates equal to 0 . Here $\rho$ and $a$ are positive, $d_{i}$ is positive for at least one value of $i$ in $A=\{1,2, \ldots\}$ and $a+\sum_{i \geq 1} d_{i}=1$. Clearly 0 is an absorbing state for the process and $A$ is an irreducible class. It is easy to establish that the chain is nonexplosive (Corollary 1 of Pollett and Taylor [10]). Brockwell [4] showed that the probability of extinction starting with $i$ individuals is 1 for all $i \in A$ if and only if $D$ (the expected increment size), given by $D=a-\sum_{i=1}^{\infty} i d_{i}=1-\sum_{i=1}^{\infty}(i+1) d_{i}$, is less than or equal to 0 ; the process is said to be subcritical, critical or supercritical according as $D$ is negative, zero or positive.

We will consider only the subcritical case. Let $d(s)=a+\sum_{i=1}^{\infty} d_{i} s^{i+1},|s|<1$, and $b(s)=d(s)-s$, so that, for example, $D=-b^{\prime}(1-)=1-d^{\prime}(1-)(<0)$. Observe that $b(0)=a(>0), b(1)=1$, that $b$ is strictly convex on $[0, \infty)$ and that $b$ has a unique zero $\sigma$ on $(0,1)$. We will study the path integral (1) with $\tau$ being the time to absorption. First we will evaluate $\mathrm{E}_{i}(\Gamma)$. We seek the minimal non-negative solution to the system

$$
i \rho a z_{i+1}-i \rho z_{i}+i \rho \sum_{j=1}^{i-1} d_{i-j} z_{j}+f_{i}=0, \quad i \geq 1 .
$$


On multiplying by $s^{i-1}$ and then summing over $i$, we find that a non-negative solution exists whenever $g(\sigma)<\infty$, where $g$ is the generating function of the sequence $\left(g_{i}, i \geq 1\right)$ given by $g_{i}=f_{i} / i: g(s)=\sum_{i=1}^{\infty} g_{i} s^{i}$. The generating function of the minimal solution is then easily evaluated in terms of $g$. We find that

$$
\sum_{i=1}^{\infty} \mathrm{E}_{i}(\Gamma) s^{i-1}=\frac{g(\sigma)-g(s)}{\rho b(s)}, \quad|s|<\sigma .
$$

Since $1 / b(s)$ has a power series expansion near $s=0$ with positive coefficients (Lemma V.12.1 of Harris [7]), we may write $1 /(\rho b(s))=\sum_{j=0}^{\infty} e_{j} s^{j},|s|<\sigma$, where $e_{j}>0$; note that $e_{0}=1 /(a \rho)$. Thus, $\mathrm{E}_{i}(\Gamma)<\infty$ if and only if $g(\sigma)<\infty$, in which case

$$
\mathrm{E}_{i}(\Gamma)=g(\sigma) e_{i-1}-\sum_{j=0}^{i-2} g_{i-1-j} e_{j}, \quad i \geq 1 .
$$

(Empty sums are taken to be 0.) To illustrate this, take $f_{i}=\alpha^{i-1}$, where $\alpha>0$, so that $g(s)=-(1 / \alpha) \log (1-\alpha s),|s|<1 / \alpha$, and hence $g(\sigma)<\infty$ provided $\alpha<1 / \sigma$. For example, if $\alpha=1$, then $f_{i}=1$ and $g(\sigma)<\infty$ (since $\sigma<1$ ). We deduce that $\mathrm{E}_{i}(\tau)<\infty$ and

$$
\mathrm{E}_{i}(\tau)=-\log (1-\sigma) e_{i-1}-\sum_{j=0}^{i-2} \frac{e_{j}}{i-1-j}, \quad i \geq 1
$$

or equivalently,

$$
\sum_{i=1}^{\infty} \mathrm{E}_{i}(\tau) s^{i-1}=\frac{1}{\rho b(s)} \log \left(\frac{1-s}{1-\sigma}\right), \quad|s|<\sigma .
$$

This is Brockwell's equation (3.1). As a further illustration, take $f_{i}=i$. In this case we have $g_{i}=1$. Hence, $g(s)=s /(1-s)$, and so $\mathrm{E}_{i}(\Gamma)=\sigma e_{i-1} /(1-\sigma)-\sum_{j=0}^{i-2} e_{j}, i \geq 1$, or equivalently,

$$
\sum_{i=1}^{\infty} \mathrm{E}_{i}(\tau) s^{i-1}=\frac{(\sigma-s)}{\rho(1-\sigma)(1-s) b(s)}, \quad|s|<\sigma .
$$

More explicit results can be obtained in the case where the catastrophe size follows a geometric law. Suppose that $d_{i}=d(1-q) q^{i-1}, i \geq 1$, where $d(>0)$ satisfies $a+d=1$, and $0 \leq q<1$. (The linear birth-death process is recovered on setting $q=0$.) It is easy to see that $D=a-d /(1-q)$. In order that $D<0$ we require $c>1$, where $c=q+d / a$. We also have

$$
b(s)=\frac{(d+q a) s^{2}-(1+q a) s+a}{1-q s}=\frac{a(1-s)(1-c s)}{1-q s},
$$

and hence $\sigma=1 / c(<1)$. The coefficients of the power series for $1 /(\rho b(s))$ are easily evaluated using partial fractions. We find that

$$
e_{j}=\frac{d c^{j}-(1-q) a}{\rho a(d-(1-q) a)}, \quad j \geq 0
$$


Thus, $\mathrm{E}_{i}(\Gamma)$ can be evaluated by substituting these expressions into (8), remembering that the expectation is finite whenever $g(\sigma)<\infty$. For example, if $f_{i}=\alpha^{i-1}$, where $\alpha>0$, the expectation will be finite when $\alpha<q+d / a$, while if $f_{i}=i$, we get

$$
\mathrm{E}_{i}(\Gamma)=\frac{q+(1-q) i}{\rho(d-(1-q) a)} .
$$

Proposition 1 can be used to identify the distribution of $\Gamma$ for specific choices of $f$. To illustrate this, suppose that $f_{i}=i$. For each $\theta>0$, we seek that maximal solution to

$$
\rho a z_{i+1}-\rho z_{i}+\rho \sum_{j=1}^{i-1} d_{i-j} z_{j}+\rho z_{0} \sum_{j=i}^{\infty} d_{j}=\theta z_{i}, \quad i \geq 1,
$$

satisfying $z_{0}=1$ and $0 \leq z_{j} \leq 1$ for $j \geq 1$. On multiplying by $s^{i-1}$ and then summing over $i$, we find that

$$
\mathrm{E}_{i}\left(e^{-\theta \Gamma}\right)=\frac{1}{1-s}-\frac{\theta(\gamma-s)}{(1-\gamma)(1-s)(\rho b(s)-\theta s)}
$$

where $\gamma=\gamma(\theta)$ is the unique solution to $\rho b(s)=\theta s$ on the interval $0<s<\sigma$. In the case of geometric catastrophes, we get

$$
\mathrm{E}_{i}\left(e^{-\theta \Gamma}\right)=\frac{\beta(\theta)-q}{1-q}(\beta(\theta))^{i-1}, \quad i \geq 1,
$$

where $\beta(\theta)$ is the smaller of the two zeros of $a \rho s^{2}-(\rho(1+q a)+\theta) s+\rho(d+q a)+q \theta$.

\section{Acknowledgements}

We would like to thank the referee for several helpful suggestions. The support of the Australian Research Council (Grant No. A00104575) is also gratefully acknowledged.

\section{References}

[1] Abate, J., Kijima, M. And Whitt, W. (1991). Decompositions of the $M / M / 1$ transition function. Queuing Systems Theory Appl. 9, 323-336.

[2] Anderson, W. (1991). Continuous-Time Markov Chains: An Applications-Oriented Approach. Springer-Verlag, New York.

[3] Ball, F. And Stefanov, V. (2001). Further approaches to computing fundamental characteristics of birth-death processes. J. Appl. Probab. 38, 995-1005.

[4] Brockwell, P. (1985). The extinction time of a birth, death and catastrophe process and of a related diffusion model. Adv. Appl. Probab. 17, 42-52.

[5] Di Crescenzo, A. (1998). First-passage-time densities and avoiding probabilities for birth-and-death processes with symmetric sample paths. J. Appl. Probab. 35, 383-394. 
[6] Flajolet, P. and Guillemin, F. (2000). The formal theory of birth-and-death processes, lattice path combinatorics and continued fractions. Adv. Appl. Probab. 32, $750-778$.

[7] Harris, T. (1963). The Theory of Branching Processes. Springer-Verlag, Berlin.

[8] Hernández-Suárez, C. M. and Castillo-Chavez, C. (1999). A basic result on the integral for birth-death Markov processes. Math. Biosci. 161, 95-104.

[9] McNeIL, D. (1970). Integral functionals of birth and death processes and related limiting distributions. Ann. Math. Statist. 41, 480-485.

[10] Pollett, P. And Taylor, P. (1993). On the problem of establishing the existence of stationary distributions for continuous-time Markov chains. Probab. Eng. Inf. Sci. 7, 529-543.

[11] Rubino, G. And Sericola, B. (1989). Sojourn times in finite Markov processes. J. Appl. Probab. 27, 744-756.

[12] Stefanov, V. And Wang, S. (2000). A note on integrals for birth-death processes. Math. Biosci. 168, 161-165.

[13] Syski, R. (1992). Passage Times for Markov Chains. IOS Press, Amsterdam. 